Tanja Žigon | Univerza v Ljubljani, Filozofska fakulteta, tanja.zigon@ff.uni-lj.si

\title{
Raumkonstruktionen aus Zentral- und Südosteuropa
}

Räumliche Semantisierungen. Raumkonstruktionen in den deutschsprachigen Literaturen aus Zentral- und Südosteuropa im 20.-21. Jahrhundert. Hg. Enikő Dácz. Regensburg: Verlag Friedrich Pustet 2018 (=Veröffentlichungen des Instituts für deutsche Kultur und Geschichte Südosteuropas an der Ludwig-Maximilians-Universität München, Bd. 135), 287 S.

In literarischen Texten ist Raum nicht nur Ort der Handlung, sondern auch kultureller Bedeutungsträger, wie Wolfgang Hallet und Birgit Neumann in ihrer 2009 herausgegebenen Monographie zum Raumbegriff in den Kulturwissenschaften feststellen. ${ }^{1}$ Der Begriff $>$ Raum $`$ ( espace $`$ ) wird bereits bei dem französischen Historiker und Kulturphilosophen Michel de Certeau (1925-1986) im Gegensatz zum Begriff `Ort $`(>l i e u<)$ dynamisch gefasst als das Resultat einer an Orten abgeleiteten Praxis, die Orte miteinander verbindet und sie in Räume individuellen Handelns verwandelt. Diese werden von unterschiedlichen Akteuren und Akteurinnen unterschiedlich dargestellt und beschrieben, im Zusammenhang mit individuellen Erfahrungswelten sowie kulturellen und räumlichen Gegebenheiten.

Vor dem Hintergrund des so genannten 'Spatial (oder auch topographical/topological) turn ‘, der in den letzten Jahrzehnten in den theoretischen Abhandlungen den $>$ Raum $`$ bzw. den geographischen Raum und räumliche Zusammenhänge und nicht mehr allein die `Zeit` in den Kultur- und Literaturwissenschaften als kulturelle Größe in den Mittelpunkt der Forschungen rückt, wird im vorliegenden Band die Poetisierung des Raumes in den deutschsprachigen Literaturen aus Zentral- und Südosteuropa im 20. Jahrhundert und danach behandelt. Dabei wird der textuelle Raum ausgewählter literarischer Texte untersucht und nach einer topographischen 
Spezifik der deutschsprachigen Literaturen des behandelten geographischen Raumes gefragt. Im Vordergrund stehen zum einen Überlegungen zur Fiktionalität des Raumes, in denen vor allem fiktionalen und realen räumlichen Gegebenheiten besondere Aufmerksamkeit gewidmet wird, zum zweiten werden semiotische Analysen der sprachlich-räumlichen Beziehungen der einzelnen Figuren untereinander und zu Gegenständen sowie zur außersprachlichen Wirklichkeit unter Heranziehung kulturwissenschaftlicher Konzepte dargestellt. Zu den thematischen Schwerpunkten gehören Strategien der Visualisierung und Spatialisierung, Inszenierung von Raumwechsel, heterotopische Raumkonstruktionen, Rauminszenierungen kultureller Begegnungen, Identitäts- und Alteritätskonzeptionen, fiktionale Wirklichkeitsmodelle der peripheren Räume wie auch Verräumlichung der Erinnerungsprozesse bzw. der Gedächtnislandschaften. Somit stehen im Fokus des Sammelbandes unterschiedliche Aspekte des Raumes in literarischen Texten, darunter der topographisch-geographische, der rhetorische, der perzeptive und der narrative Gesichtspunkt. Thematisiert werden sowohl die sogenannten >Regionalliteraturen « wie auch Werke von Autoren und Autorinnen mit zentral- oder südosteuropäischem Migrationshintergrund.

Der Band, eröffnet mit einem längeren Vorwort der Herausgeberin Enikő Dácz, versammelt 16 Beiträge, die 2015 im Rahmen der vom Institut für deutsche Kultur und Geschichte Südosteuropas an der Ludwig-Maximilians-Universität München (IKGS) organisierten Sektion zum Thema »Raumkonstruktionen in den deutschsprachigen Literaturen in und aus Ostmittel- und Südosteuropa« am X. Internationalen Kongress der Germanisten Rumäniens in Kronstadt (rum. Braşov, ung. Brassó) präsentiert wurden. Im Titel des Sammelbandes wurde in Anlehnung an Moritz Csáky lediglich der Begriff Mitteleuropa durch das unproblematischere Zentraleuropa ersetzt. Die Verfasser kommen aus Deutschland, Kroatien, Slowenien, Rumänien, Ungarn und aus den Vereinigten Staaten. Die Beiträge sind in zwei umfassende Kapitel eingeteilt, und zwar werden im ersten fiktionale Räume, im zweiten die Rauminszenierungen in medialen Kontexten (Essays, Autobiographien, Memoiren, Zeitungen, Korrespondenzen) besprochen. In der thematischen Vielfalt der Beiträge spiegelt sich der derzeitige Forschungsstand zur zeitgenössischen Raumperzeption und -gestaltung in den deutschsprachigen Literaturen aus Zentral- und Südosteuropa.

Hervorzuheben ist die methodologische und thematische Stringenz der Beiträge, die allesamt analytische Schärfe, überzeugende wissenschaftliche Argumentation und kritisch ausgewogene Urteile sowie eine transparente Vermittlung aufweisen. Das zentrale Thema des Bandes wird in der einleitenden Studie von Magdolna Orosz " Raum` und >Raumdarstellung` als 
Kategorien literarischer (narrativer) Textanalyse« erörtert. Der Aufsatz bietet einen konzisen Überblick zum Kanon der kultur- und literaturwissenschaftlichen Raumtheorie und -forschung und führt darüber hinaus vier kurze Fallbeispiele an, die sich auf Österreich-Ungarn beim Ausbruch des Ersten Weltkrieges beziehen und regionalspezifische Momente der Raumkonstruktion sowie deren historische Bezüge erschließen. Die hier geschilderten Gemeinsamkeiten der Raumnarrative kommen auch in den beiden thematisch und chronologisch strukturierten Teilen des Sammelbandes deutlich zum Ausdruck.

Im ersten Teil des Bandes - „Fiktionale Räume« - sind sieben Beiträge versammelt. Enikő Dácz knüpft an Orosz an und untersucht unter Berücksichtigung narratologischer und kulturwissenschaftlicher Ansätze die literarische Raumgestaltung im Roman Zwischen Grenzen und Zeiten, verfasst von dem wegen seiner kulturpolitischen Ansichten umstrittenen siebenbürgisch-sächsischen Autor Heinrich Zillich (1898-1988). Im Vordergrund steht die Frage, in welchem Maße der ideologisch engagierte Roman " performativ den Raum mitkonstruiert, den [er] repräsentiert « (S. 40), also Siebenbürgen in der Zeit während des und gleich nach dem Ersten Weltkrieg. - Im nächsten Aufsatz versucht die Germanistin und Rumänistin Laura Laza anhand des Nachlasses von Wolf von Aichelburg (1912-1994), der sich im Archiv des Nationalen Rats für das Studium der Archive der Securitate befindet, den fiktionalen Raum in seiner Lyrik zu analysieren. Die in rumänischen Gefängnissen und Arbeitslagern entstandenen Gedichte des 1912 im heute kroatischen Pula (Pola) geborenen Dichters, dessen Familie sich 1918 im siebenbürgischen Hermannstadt (rum. Sibiu) niederließ, waren bis dato nicht veröffentlicht. Die Autorin geht in ihrem Beitrag auf die Frage ein, wie sich das "räumliche Imaginäre (S. 55) in den Gedichten Aichelburgs über den realen Raum legt. - Ausgehend von einer ähnlichen Fragestellung werden in dem Beitrag von Raluca Cernahoschi die realen und imaginären Topographien in dem Poem La Victoire von Horst Samson (1954) analysiert. Obwohl es sich bei den Schauplätzen in den 52 Teilgedichten um rumänische Orte handelt, kann trotzdem nicht von einem einheitlichen Raum >Rumänien` gesprochen werden. In Anlehnung an Andrea Mahlers Theorie analysiert Cernahoschi das Verhältnis von erfahrenem und literarischem Raum und stellt fest, dass es sich um eine literarische Topographie handelt, in der sich dieselben geographischen Orte in äußerst verschiedene (Text-)Räume verwandeln. Es werden Transit-, Macht- und Gegenräume untersucht, darunter apokalyptische Räume des Dorfes der Kriegszeit, Räume der Deportation und Räume der Auswanderung vieler Rumäniendeutscher in der Zeit des kommunistischen Regimes, wobei auf 
die Frage nach der Schaffung eines weltliterarischen interkontextualen Raumes als Befreiungsstrategie der Gedichte eingegangen wird. Der Zug, der die Räume in dem Langgedicht verbindet, ist in diesem Sinne, wie die Autorin feststellt, eine Heterotopie, ein bewegliches »Stück Raum «, »ein Ort ohne Ort« (S. 81), der der Landschaft ausgeliefert ist, eine räumlich (re) konstruierte Geschichte der Banater Schwaben. - Diese ist auch das zentrale Thema auch des nächsten, von Roxana Nubert und Ana-Maria DascăluRomiţan verfassten Beitrags, in dem das Banat als Erinnerungsraum von dem in Österreich lebenden rumäniendeutschen Lyriker und Erzähler Johann Lippet (1951) analysiert wird. Auf der Suche nach einer verlorenen Zeit und einem verlorenen Land schildert Lippet in seinen Texten fiktive Untergangsszenarien der Banater Schwaben und reflektiert persönliche und historische Prozesse, wobei die Geschichte einzelner Familien mit der der Gemeinschaft verschmilzt. - Die ungarische Literaturwissenschaftlerin und Komparatistin Eszter Propszt widmet sich in ihrer Analyse der Raumkonstruktion als Identitätskonstruktion im Roman Dort drüben von Béla Bayer, in dem die ungarndeutsche Geschichte als ein Raummodell dargestellt wird. Die Untersuchung orientiert sich an dem semiotischen Raumkonzept von Jurij Lotman und geht der Frage nach, was für ein Identifikationsangebot des Raummodells dem Leser zur Verfügung steht sowie ob und wie damit die alltäglichen und historischen Erfahrungen der Ungarndeutschen interpretiert werden können.

Die letzten zwei Beiträge im ersten Teil des Sammelbandes behandeln spezifische Themen von Autoren mit Migrationshintergrund, darunter vor allem ihre Verortung zwischen den Sprachen und Räumen. Raluca Rădulescu setzt sich mit dem literarischen Opus der aus Kroatien stammenden Dragica Rajčić (1959) auseinander, erfasst vor theoretischem Hintergrund (Michel Foucault, Marc Augé, Jürgen Habermas) die vielfache identitäre Verortung ihrer Texte als Poetik des Raumes und kommt zu dem Schluss, dass Rajčićs Heimat ein Nicht-Ort sei, wo es keine Grenze zwischen Realität und Fantasie gebe. - Mit einer ähnlichen Thematik wird der Leser auch im Beitrag von Réka Sánta-Jakabházi konfrontiert: Im analysierten Roman von Aglaya Veteranyi (1962-2002), in dem eine aus Rumänien geflüchtete Artistenfamilie im Mittelpunkt steht, wird der Zirkus als heterotopischer Raum dargestellt.

Den zweiten Teil des Sammelbandes, der sich den Rauminszenierungen in medialen Kontexten widmet, eröffnet der Beitrag des rumänischen Germanisten Szabolcs János. Darin werden die regionalen Kulturen Siebenbürgens und des Banats in den Reiseberichten des 18. und 19. Jahrhunderts besprochen, wobei die Konstruiertheit der Räume wie auch die problematische 
Frage nach den sog. Regionalliteraturen und deren Rolle beim Entstehen der >regionalen Identitäten im Vordergrund stehen. - Olivia Spiridon knüpft im folgenden Aufsatz an János an und setzt sich mit dem Aspekt der Grenze in der Banater Gemeinde Hatzfeld im 20. Jahrhundert auseinander. Das Grenzgebiet, der Lebensraum der Deutschen in Rumänien, wird als Raum der Konflikte, Eskalationen, aber auch Kompromisse dargestellt, wo es zu einem regen Austausch und innovativen Überschreitungen kommt. - Während Spiridon wie auch Dácz im ersten Teil des Sammelbandes die Verbindung von Raumgestaltung und Ideologie thematisieren, interessieren im nächsten Beitrag von Michaela Nowotnick literarische Wechselbeziehungen zwischen den Räumen im geographischen Sinn, nämlich zwischen Siebenbürgen und Deutschland; die literarischen Verflechtungen werden am Beispiel der Zeitschrift »Ostland « und deren Herausgebers Richard Csaki interpretiert. - Die folgenden drei Studien widmen sich einer fernen Provinz, der Bukowina. Ana-Maria Pălimariu bespricht anhand der Autobiografie des Psychiaters und Psychoanalytikers Wilhelm Reich (1897-1957) das imaginäre Gesellschaftsbild der Bukowiner Juden und dekonstruiert anhand der analysierten Autobiografie den Mythos vom Westen und Osten. - In der folgenden Abhandlung, verfasst von George Guţu, wird anhand der Korrespondenzen von Alfred Margul-Sperber (1898-1967) mit Bukowiner Autoren (Moses Rosenkranz, Rose Ausländer und Paul Celan) der Untergang vom Bukowina-Mythos mit seinen Konsequenzen nachgezeichnet; der Raum erscheint hier als Inszenierung, die notwendig ist, um dichterische Identität zu erhalten. - Der dritte Beitrag, der im selben geographischen Raum verbleibt, setzt sich mit dem Erzählungsband Sie trommelten mit den Fäusten den Takt von Edgar Hilsenrath (1926) auseinander. Francisca Solomon untersucht darin die Bukowina und Transnistrien als Gedächtnislandschaft der Katastrophe, wobei sie auf theoretische Forschungsergebnisse zu Erinnerungsorten von Aleida Assmann und die Theorie der Mechanismen jüdischer Identitätskonstruktion von James Young Bezug nimmt.

Im Fokus der letzten zwei Aufsätze steht der südslawische Raum. Die Zagreber Germanistin Milka Car thematisiert die Verflechtung von politischer Ideologie und literarischer Raumgestaltung am Beispiel des berühmten Essays Illyricum sacrum von Miroslav Krleža (1893-1981), während sich Irena Samide (Ljubljana) mit den Lebens-, Schrift-, Geschlechter- sowie geobiographischen Räumen in den Reiseberichten der bekannten Autorin und Grenzgängerin Alma M. Karlin (1889-1950) auseinander setzt.

Abschließend sei hervorgehoben, dass die Stärke des Sammelbandes in der Anregung für mehrere Bereiche literatur- und kulturwissenschaftlicher Forschung liegt: Der Band bietet nicht nur einen neuen, wichtigen Anstoß 
zur Diskussion über die Raumkonstruktionen in der deutschsprachigen Literatur, sondern untersucht anhand einer Reihe von Fallbeispielen Zentral- und Südosteuropa als textuellen Raum in literarischen Texten, Reiseberichten, Autobiografien, Essays oder Korrespondenzen; es gewährt aber auch einen Einblick in die Rolle der Übersetzung, denn der untersuchte Text von Krleža Illyricum sacrum liegt in deutschsprachiger Übersetzung vor, wurde jedoch ursprünglich auf Kroatisch verfasst. Insgesamt ist der Sammelband auch als Anregung für weitere interkulturelle und interdisziplinäre Analysen zu verstehen, ein Anstoß für weitere Untersuchungen räumlicher Narrative aus komparatistischer Perspektive. 